\title{
Multi-stage production with variable lot sizes and transportation of partial lots
}

\author{
Z. DREZNER \\ School of Management, University of Michigan-Dearborn, Dearborn, MI 48128, U.S.A.
}

\author{
A.Z. SZENDROVITS and G.O. WESOLOWSKY \\ Faculty of Business, McMaster University, Hamilton, Ontario, Canada L8S $4 M 4$
}

Received November 1982

Revised March 1983

This paper describes a model for a multi-stage production/inventory system where lots may be of different sizes. In addition, either completed lots or partial lots, called batches, may be transported to succeeding stages. The model incorporates constraints on lot and batch-sizes and thus provides a rather comprehensive set of possibilities for organizing a production/inventory system. A heuristic solution procedure is developed and is shown to be 'close to optimal' by bounding.

\section{Introduction}

When the rate of continuous demand is smaller than the manufacturing rate for a product, intermittent manufacturing in economic lot-sizes is usually justified. Economic lot-sizes are also important when the continuous demand of an assembly line is fed by a part which is manufactured intermittently. The single-stage lot-size model is frequently misused in multi-stage manufacturing because it ignores the work-in-process inventory and overstates the optimal lot-size.

Lot-size models impose a constraint on the scheduling of production facilities which are shared by several products because the manufacturing of lots must be scheduled with priority. Facility scheduling is a lesser problem in single-stage production models than in multi-stage models. Even in the multi-stage case, if a relatively small portion of all products have scheduling priority, there is ample room for manipulating the schedule of the rest of the product line. The key problem is to identify those products which constitute a substantial part of the work-in-process and represent a relatively small portion of the total production capacity. If such products are scheduled according to an appropriate lot-size model, the process inventory and the total inventory cost can be reduced considerably.

The state of the art of production/inventory research is well summarized in a recent comprehensive survey of the literature [10]. Since the mid 70's multi-stage production/inventory models have gained increasing attention. The terminology used in the literature varies substantially. In this paper we call a quantity produced with one set-up at a stage a 'lot' and a portion of a lot transported to the next stage a 'batch'. Deterministic lot-size models for multi-stage serial and assembly systems represent a variety of process organizations. Two classes of these models, both based on an infinite time horizon, can be distinguished in the literature. One class, which we call 'variable lot-size models' $[1,2,5,9]$, allows different and non-increasing lot-sizes across stages. Only complete lots are transported to the next stage and the lot-size of a stage is an integer multiple of the lot-size that follows it. One variable lot-size model [4] does not have integrality restrictions; it is analytically tractable because any portion of a lot can be transported

This research was supported by a grant from the Natural Sciences and Engineering Reserach Council of Canada.

North-Holland

European Journal of Operational Research 17 (1984) 227-237 
to the next stage at zero cost. Another class, which we call 'batch shipment models' $[6,7,8]$, has uniform lot-sizes at all stages but allows portions of a lot to be transported to the next stage is equal-sized batches at some cost per batch.

In this paper, we present a lot-size model for a single product that is manufactured in a serial system through a large number of stages. The model and its assumptions are briefly summarized as follows:

(a) Lot-sizes can be variable and subject to constraints, but must be integer multiples of the lot-size at the following stage. Starting a new lot involves a fixed set-up cost.

(b) Either complete lots or equal-sized batches can be shipped to the next stage at a transportation cost for each shipment. The unit cost of transportation is related to the load capacity of the transport equipment.

(c) Lot sizes and batch-sizes are not restricted to integers. Set-up times of lots and transportation times of batches are assumed to be negligible.

(d) Deterministic (constant) production and demand rates are assumed over an infinite horizon. No backlogging (deliberate shortage) of inventory is permitted in the system.

(e) Linear inventory-holding costs are assumed at all stages. The cost of holding one unit of process inventory is related to the stage which has been completed and cannot decrease at the following stage (due to the added value of the product).

Our model combines the features of the variable lot-size and the batch shipment models and uses the conventional assumptions in the literature. The use of variable lot-sizes balances multiple set-up costs at some stages against the decreased size and cost of the process inventory. Transporting batches instead of complete lots may result in higher transportation costs. On the other hand, production at subsequent stages might be scheduled with overlap on the same lot to further reduce the size and the cost of the average process inventory. As an additional element of flexibility beyond that in existing models, our model can accommodate constraints on lot-sizes that may result from limited production or storage capacity as well as constraints on batch-sizes that may be caused by limited load-capacity of the transport equipment.

Usually, our model would yield a lower (certainly never higher) cost than either of the variable lot-size or the batch shipment models. Hence, it is an economical substitute for these models whenever they are applicable to a serial production system. This is certainly the case in the automobile and generally in the machine industry where many parts of a complex product involve a series and usually large number of production operations (stages). Part manufacturing is seldom synchronized with the rhythm of the assembly line; therefore, a single part is produced in lots intermittently. While a single part may not be sold directly, the assembly of the product incorporating this part could create a continuous demand over time. This is the reason for the applicability of these lot-size models.

Although the generalizations of previous models that are presented in this paper are straightforward, the add considerable realism to the representation of the process organization. At the same time, they increase substantially the difficulty of solving a traditionally formidable optimization problem.

\section{Constructing the cost function}

First, we define our notation. The stages in the production system are $i=1,2, \ldots, n$; the final stage, the one which meets the demand for the finished product, is stage 1. Other symbols are as follows:

$D=$ demand (consumption) rate of the final product (at stage 1),

$P_{i}=$ production rate at stage $i$ (note that $P_{i}>D$ ),

$F_{i}=$ fixed (set-up) cost per lot at stage $i$,

$T_{i}=$ transportation cost of one load from stage $i$ to the next,

$g_{i}=$ the maximum size of one load (load capacity) transported from the $i$ th stage to the next,

$c_{i}=$ unit inventory-holding cost per unit time, at stage $i$,

$Q_{i}=$ the lot-size at stage $i$,

$L_{i}=$ the maximum lot size permitted at stage $i$, 


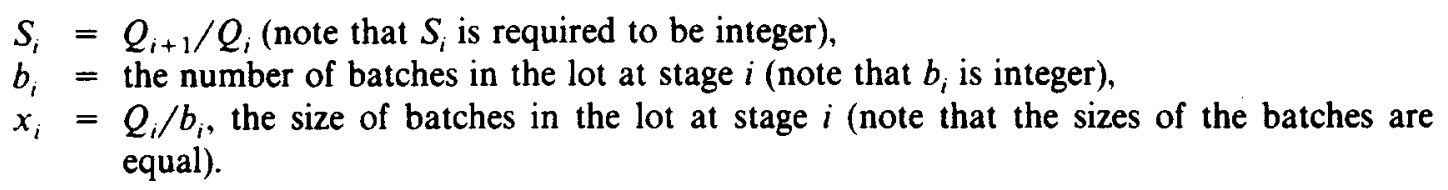

All parameters above are greater than zero. Also, it should be noted that

(i) a non-integer value, $A$, 'rounded up' to the nearest integer is denoted by $|A|$,

(ii) the 'rounded-down' value is denoted by $\lfloor A\rfloor$,

(iii) $[\lfloor A\rfloor]$ denotes conventional rounding to the nearest integer,

(iv) an integer rounded is the integer.

For the convenience of our equations we define

$$
Q_{0}=Q_{1}, \quad c_{n+1}=0, \quad P_{n+1}=0, \quad P_{0}=D, \quad S_{n}=1 .
$$

To derive the cost function, we start by examining Fig. 1 which illustrates the production/inventory model of two adjacent stages $i$ and $i-1$ for the case when $P_{i}<P_{i-1}$. The upper dashed line with slope $P_{i}$ shows the uninterrupted production build-up of lot-size $Q_{i}$ at stage $i$. From this stage $b_{i}$ number of equal-sized batches, each of size $x_{i}=Q_{i} / b_{i}$, are transported to the next stage, $i-1$. The rectangular vertex of the triangle (the sides of which form a step function) immediately below the dashed line represents the point at which each batch is available for production at stage $i-1$. The other step function with slope $P_{i-1}$ shows the production build-up of $Q_{i-1}$ lot sizes at stage $i-1$. The dashed line below this step function, the slope of which is $D$, indicates the cumulative demand which must be matched by the cumulative production at every stage. It can be seen that the inventory at stage $i-1$ cycles back to zero every $Q_{i-1} / D$ units of time.

Let $R_{i-1}$ be the earliest possible time for the start of production at stage $i-1$. To find an analytical expression for $R_{i-1}$ we denote the sequence of batches shipped from stage $i$ with $j=1,2, \ldots, b_{i}$.

Clearly, we wish to start producing at stage $i-1$ as soon as possible to minimize the size of the process inventory, but $R_{i-1}$ is constrained by the point where the step functions touch. As can be seen in Fig. 1 this happens when $j+1=6$ batches are completed at stage $i$ during $(j+1) x_{i} / P_{i}$ units of time. During this time $\left\lfloor j x_{i} / Q_{i-1}\right\rfloor=2$ lots are completed at stage $i-1$ requiring $\left\lfloor j x_{i} / Q_{i-1}\right\rfloor Q_{i-1} / P_{i-1}$ units of time. These lots

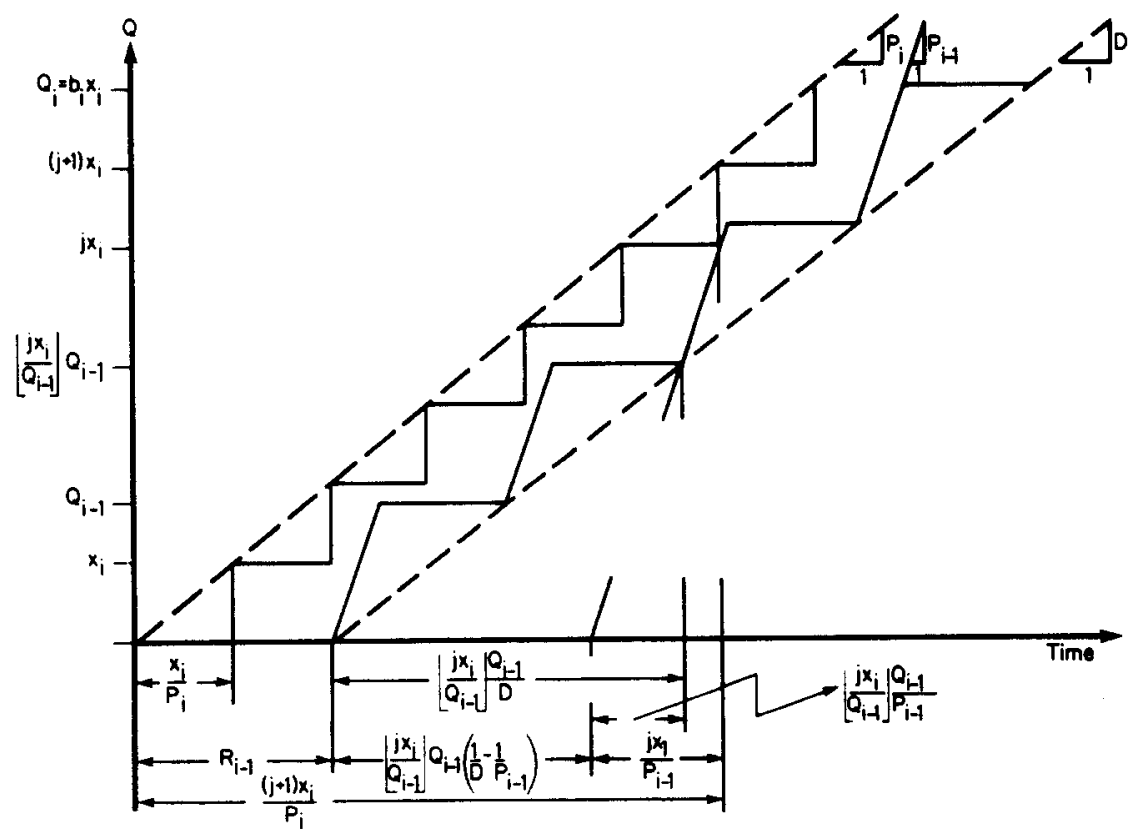

Fig. 1. Build-up of inventory between adjacent stages when $P_{i}<P_{i-1}$. 
satisfy demand during $\left\lfloor j x_{i} / Q_{i-1}\right\rfloor Q_{i-1} / D$ time periods. It is easy to verify that $j x_{i} / P_{i-1}$ units of time are needed to produce $j x_{i}$ units at stage $i-1$. From these distances on the time scale we find the following analytical expression:

$$
R_{i-1}=(j+1) x_{i} / P_{i}-j x_{i} / P_{i-1}-\left\lfloor j_{x_{i}} / Q_{i-1}\right\rfloor Q_{i-1}\left(1 / D-1 / P_{i-1}\right) .
$$

Therefore, as is seen in Fig. 1, to keep stage $i-1$ production supplied it must be true that

$$
R_{i-1}+j x_{i} / P_{i-1}+\left\lfloor j x_{i} / Q_{i-1}\right\rfloor Q_{i-1}\left(1 / D-1 / P_{i-1}\right) \geqslant(j+1) x_{i} / P_{i} \text { for } 0 \leqslant j \leqslant b_{i}-1 .
$$

Of course, this will be true if

$$
R_{i-1}=\max _{0 \leqslant j \leqslant b_{1}-1}\left\{(j+1) x_{i} / P_{i}-j x_{i} / P_{i-1}-\left\lfloor j x_{i} / Q_{i-1}\right\rfloor Q_{i-1}\left(1 / D-1 / P_{i-1}\right)\right\} .
$$

Rearranging the expression for $R_{i-1}$ we obtain

$$
R_{i-1}=x_{i} / P_{i}+\max _{0 \leqslant j \leqslant b_{i}-1}\{\theta(j)\}
$$

where

$$
\begin{aligned}
\theta(j) & =j x_{i}\left(1 / P_{i}-1 / P_{i-1}\right)-\left\lfloor j x_{i} / Q_{i-1}\right\rfloor Q_{i-1}\left(1 / D-1 / P_{i-1}\right), \\
j & =0,1, \ldots, b_{i}-1 \text { and } j=\text { integer. }
\end{aligned}
$$

One could illustrate graphically that if $P_{i} \geqslant P_{i-1}$, stage $i-1$ can be started as soon as the first batch is completed at stage $i$, which is after $x_{i} / P_{i}$ units of time. It is also easy to verify that expression (1) is also valid for this case. When $P_{i} \geqslant P_{i-1}$ the quantity $\left(1 / P_{i}-1 / P_{i-1}\right)$ is non-positive and $j=0$ provides the maximum in (1); thus

$$
R_{i-1}=x_{i} / P_{i} \text { for } P_{i} \geqslant P_{i-1} \text {. }
$$

It will be useful to examine the lower bound on $R_{i-1}$. Naturally, in the case above the lower bound on $R_{i-1}$ is

$$
\hat{R}_{i-1}=x_{i} / P_{i} \text { for } P_{i} \geqslant P_{i-1} \text {. }
$$

It is also obvious from Fig. 1 that if $P_{i}<P_{i-1}$ the shortest time for $R_{i-1}$ will occur when the step functions touch during the production of the first lot at stage $i-1$. That is when $j=\left(\left[Q_{i-1} / x_{i}\right]-1\right)$. Substituting this for $j$ in (1) we obtain the lower bound on $R_{i-1}$ which is

$$
\hat{R}_{i-1}=x_{i} / P_{i}+\theta\left(\left[Q_{i-1} / x_{i}\right]-1\right) \text { for } P_{i}<P_{i-1} \text {. }
$$

Since part of $\theta(j)$ in $(1)$ is $\left\lfloor j x_{i} / Q_{i-1}\right\rfloor$ which becomes $\left.\left\lfloor\left(\mid Q_{i-1} / x_{i}\right\rfloor-1\right) x_{i} / Q_{i-1}\right\rfloor=0$, the analytical expression for the lower bound on $R_{i-1}$ is

$$
\hat{R}_{i-1}=x_{i} / P_{i}+\left(\left|Q_{i-1} / x_{i}\right|-1\right) x_{i}\left(1 / P_{i}-1 / P_{i-1}\right) \text { for } P_{i}<P_{i-1} \text {. }
$$

The two lower bounds can be combined in one general expression for $\hat{R}_{i-1}$ :

$$
\hat{R}_{i-1}=x_{i}\left[1 / P_{i}+\left(l_{i}-1\right)\left(1 / P_{i}-1 / P_{i-1}\right)\right]
$$

where

$$
l_{i}= \begin{cases}1 & \text { if } P_{i} \geqslant P_{i-1} \\ \left\lceil Q_{i-1} / x_{i}\right] & \text { if } P_{i}<P_{i-1}\end{cases}
$$

Actually, this rather than the case in Fig. 1 is usual. $R_{i-1}$ is not equal to $\hat{R}_{i-1}$ only if $D$ is 'nearly equal' to $P_{i}$. Fig. 2.

Having found the analytical expression for $R_{i-1}$ we can determine the inventory at stage $i$ as shown in 


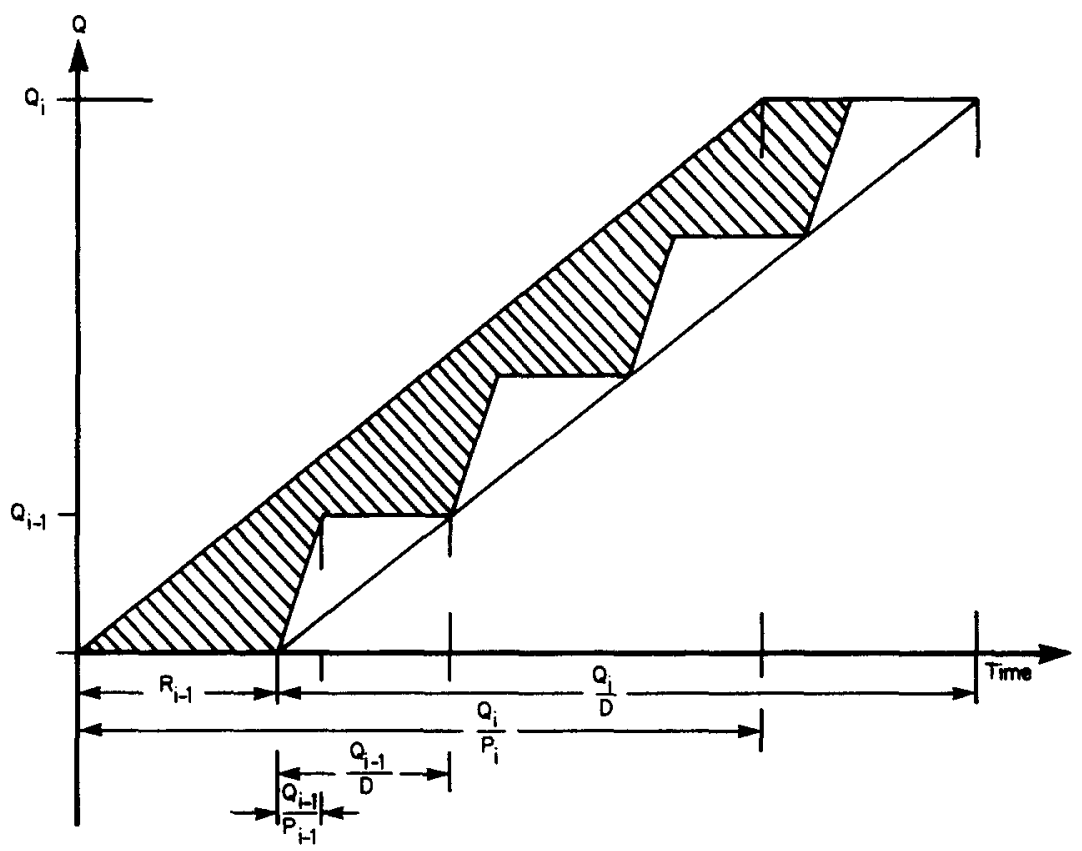

Fig. 2. Time-weighted inventory of stage $i$.

To find the average inventory-holding cost at stage $i$, we start by finding the time-weighted inventory (shaded area in Fig. 2). This is done by subtracting triangles from a trapezium. Then, we divide the area by $Q_{i} / D$ to obtain the average inventory and multiply by $c_{\text {, }}$ to obtain the inventory-holding cost per unit time. Thus $C_{i}$, the average inventory-holding cost of stage $i$ is

$$
C_{i}=\frac{1}{2} c_{i} D\left[2 R_{i-1}+Q_{i}\left(1 / D-1 / P_{i}\right)-Q_{i-1}\left(1 / D-1 / P_{i-1}\right)\right] .
$$

Since $P_{0}=D$ the expression for $C_{i}$ holds for all stages.

It is interesting to consider the possibility of using batch-sizes that are larger than the load capacity of the transport equipment, $x_{i}>g_{i}$. Each batch would thus require $\left[x_{i} / g_{i}\right]$ loads and hence the transportation cost per $Q_{i}$ lot-size would be $\left.T_{i} \mid Q_{i} / x_{i}\right]\left|x_{i} / g_{i}\right|$. If batch-sizes are limited to $x_{i} \leqslant g_{i}$, the transport cost per $Q_{i}$ lot-size would be $T_{i}\left[Q_{i} / g_{i}\right]$ which is obviously smaller. It also can be verified, by examining Fig. 1 , that increasing the number of batches and hence decreasing the batch-size involves a smaller $R_{i-1}$ (earlier start of production at stage $i-1)$ and thus saves inventory-holding cost. The constraint $x_{i} \leqslant g_{i}$ allows us to simplify the statement of the cost function.

Recall that $F_{i}$ is the set-up cost per lot at stage $i$. The sum of the set-up and transportation costs, $\left(F_{i}+b_{i} T_{i}\right)$, divided by $Q_{i} / D$ gives the average fixed cost per unit time.

The total cost of the system can be obtained by summing the fixed and inventory-holding costs:

$$
\mathrm{TC}=D \sum_{i=1}^{n}\left\{\left(F_{i}+b_{i} T_{i}\right) / Q_{i}+\frac{1}{2} c_{i}\left[2 R_{i-1}+Q_{i}\left(1 / D-1 / P_{i}\right)-Q_{i-1}\left(1 / D-1 / P_{i-1}\right)\right]\right\} .
$$

Since $c_{n+1}=0$, we can rearrange (4) and express the total cost in terms of $Q_{i}$. Thus, the optimization problem is as follows:

$$
\begin{array}{lll}
\text { minimize } & \mathrm{TC}=D \sum_{i=1}^{n}\left\{F_{i} / Q_{i}+Q_{i}\left(1 / D-1 / P_{i}\right)\left(c_{i}-c_{i+1}\right) / 2+c_{i} R_{i-1}+T_{i} / x_{i}\right\}, \\
\text { subject to } & x_{i} \leqslant g_{i} & \text { for } i=1, \ldots, n, \\
& Q_{i} \leqslant L_{i} & \text { for } i=1, \ldots, n, \\
& Q_{i+1} / Q_{i}=S_{i}=\text { positive integer } & \text { for } i=1, \ldots, n-1, \\
& Q_{i} / x_{i}=b_{i}=\text { positive integer } & \text { for } i=1, \ldots, n .
\end{array}
$$




\section{Bounding}

In order to obtain a lower bound on the cost of the optimal solution to problem (5) we relax the integrality constraints on $S_{i}, l_{i}$ and $b_{i}$, replace $R_{i-1}$ with $\hat{R}_{i-1}$ and solve the problem using $x_{i}=Q_{i} / b_{i}$. Thus we have

$$
l_{i}^{\mathrm{c}}= \begin{cases}1 & \text { if } P_{i} \geqslant P_{i-1} \\ Q_{i-1} / x_{i} & \text { if } P_{i}<P_{i-1}\end{cases}
$$

Hence

$$
\hat{R}_{i-1}^{\mathrm{c}}=x_{i}\left[1 / P_{i}+\left(l_{i}^{\mathrm{c}}-1\right)\left(1 / P_{i}-1 / P_{i-1}\right)\right] .
$$

Now, we introduce a new indicator variable

$$
\delta_{i}= \begin{cases}0 & \text { if } P_{i} \geqslant P_{i-1} \text { or } i=n+1 \\ 1 & \text { otherwise }\end{cases}
$$

which we can use in (7); therefore

$$
\hat{R}_{i-1}^{\mathrm{c}}=\left(1-\delta_{i}\right) x_{i} / P_{i}+\delta_{i}\left[x_{i} / P_{i-1}+Q_{i-1}\left(1 / P_{i}-1 / P_{i-1}\right)\right] .
$$

Substituting (9) into (5) and letting $\delta_{i+1}=0$, we obtain the following total cost expressed in terms of $Q_{\text {, }}$ and $x_{i}$ :

$$
\begin{aligned}
\mathrm{TC}^{\mathrm{c}}= & D \sum_{i=1}^{n}\left\{F_{i} / Q_{i}+Q_{i}\left[\left(1 / D-1 / P_{i}\right)\left(c_{i}-c_{i+1}\right) / 2+\delta_{i+1} c_{i}\left(1 / P_{i+1}-1 / P_{i}\right)\right]\right. \\
& \left.+x_{i} c_{i}\left[\left(1-\delta_{i}\right) / P_{i}+\delta_{i} / P_{i-1}\right]+T_{i} / x_{i}\right\} .
\end{aligned}
$$

This can be written as

$$
\mathrm{TC}^{\mathrm{c}}=D \sum_{i=1}^{n}\left\{A_{i} / Q_{i}+B_{i} Q_{i}+H_{i} x_{i}+G_{i} / x_{i}\right\}
$$

where the $A_{i}, B_{i}, H_{i}$ and $G_{i}$ coefficients are evident from (10). Since $\hat{R}_{i-1}^{\mathrm{c}} \leqslant \hat{R}_{i-1}, \mathrm{TC} \leqslant \mathrm{TC}$ for all values of $Q_{i}$ and $x_{i}$, now we have the problem

$$
\begin{array}{ll}
\text { minimize } & \mathrm{TC}^{\mathrm{c}}, \\
\text { subject to } & Q_{i} \leqslant L_{i}, \\
& x_{i} \leqslant g_{i}, \\
& Q_{i-1} \leqslant Q_{i}, \\
& x_{i} \leqslant Q_{i}, \\
& Q_{i}, x_{i} \geqslant 0 .
\end{array}
$$

The solution to this problem provides the lower bound on the solution to (5). Since $\mathrm{TC}^{\mathrm{c}}$ is convex, as can be seen from (11), we could use nonlinear programming. An efficient method is given in [3].

\section{A heuristic solution procedure}

First, we find integers $S_{i}$ by the following cumulative rounding procedure. Let $Q_{1}^{\mathrm{c}}, \ldots, Q_{n}^{\mathrm{c}}$ be the optimal $Q_{i}$ values found in problem (12); then the $S_{i}$ 's are determined in sequence by

$$
S_{k}=\left[\left\lfloor\frac{Q_{k+1}^{\mathrm{c}}}{Q_{i}^{\mathrm{c}}\left(S_{1} S_{2} \cdots S_{k-1}\right)}\right\rfloor \mid \text { for } k=1, \ldots, n-1\right.
$$

where $S_{0}=1$. 
Since $L_{i} /\left(S_{1} S_{2} \cdots S_{i-1}\right)$ is the maximum allowable $Q_{1}$, given the lot-size constraint $L_{i}$, at stage $i$,

$$
Q_{1 \mathrm{u}}=\min _{1 \leqslant i \leqslant n}\left\{L_{i} /\left(S_{1} S_{2} \cdots S_{i-1}\right)\right\}
$$

is the upper bound for $Q_{1}$. It can be shown that $Q_{1} \leqslant Q_{1 u}$ if and only if $Q_{i} \leqslant L_{i}$ for $i=1, \ldots, n$. Therefore we choose

$$
Q_{i}=\min \left\{Q_{1 \mathrm{u}}, Q_{1}^{\mathrm{c}}\right\}, \quad Q_{i}=Q_{1} S_{1} \cdots S_{i-1} .
$$

Next, we determine the best integer $b_{i}$ 's for the $Q_{i}$ 's found in (15). First, we consider the case where $R_{i-1}=\hat{R}_{i-1}$. In (5) we convert each $x_{i}$ to $Q_{i} / b_{i}$; hence each term of the sum now containing $b_{i}$ can be optimized separately with respect to $b_{i}$. Terms not containing $b_{i}$ (constants) are ignored and we have $n$ problems of the type

$$
\text { minimize } f\left(b_{i}\right)=\left(T_{i} / Q_{i}\right) b_{i}+c_{i} \hat{R}_{i-1} .
$$

Substituting (2) this can be written as follows:

$$
\begin{array}{ll}
\text { minimize } & f\left(b_{i}\right)=\left(T_{i} / Q_{i}\right) b_{i}+\left(Q_{i} / b_{i}\right) c_{i}\left[1 / P_{i}+\left(l_{i}-1\right)\left(1 / P_{i}-1 / P_{i-1}\right)\right], \\
\text { subject to } & b_{i} \geqslant Q_{i} / g_{i},
\end{array}
$$

where

$$
l_{i}= \begin{cases}1 & \text { if } P_{i} \geqslant P_{i-1} \\ \left\lceil b_{i} / S_{i}\right] & \text { if } P_{i}<P_{i-1}\end{cases}
$$

If $P_{i} \geqslant P_{i-1}$, then $l_{i}=1$ and $f\left(b_{i}\right)$ is convex. We find the lowest cost integer on either side of the non-integer solution $b_{i}=Q_{i} /\left(P_{i} T_{i}\right)^{1 / 2}$. Then, we compare this integer with $\left|Q_{i} / g_{i}\right|$ and take the larger of the two.

If $P_{i}<P_{i-1}$, the problem is more difficult. In this case,

$$
f\left(b_{i}\right)=\left(T_{i} / Q_{i}\right) b_{i}+\left(Q_{i} / b_{i}\right) c_{i}\left[1 / P_{i}+\left(r_{i}+b_{i} / S_{i}-1\right)\left(1 / P_{i}-1 / P_{i-1}\right)\right]
$$

where $r_{i}=\left\lceil b_{i} / S_{i}\right\rceil-b_{i} / S_{i}$ and $0 \leqslant r_{i} \leqslant 1$.

Let $y_{i}=b_{i} / S_{i}$, and consider the function

$$
h\left(y_{i}, r_{i}\right)=\left(u_{i}+w_{i} r_{i}\right) / y_{i}+v_{i} y_{i}+w_{i}
$$

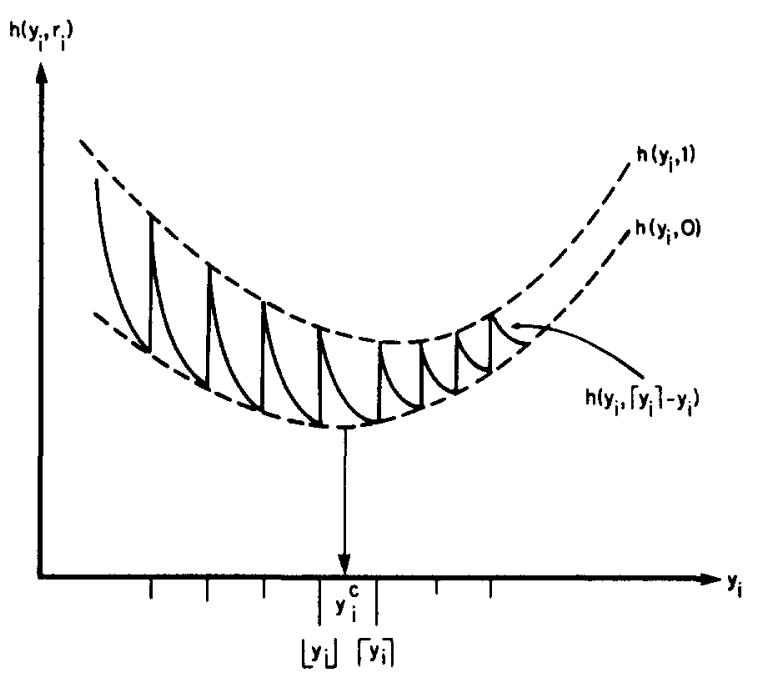

Fig. 3. Illustration of a 'typical' function $h\left(y_{i}, r_{i}\right)$ in Eq. (18). 
where

$$
u_{i}=\left(Q_{i} / S_{i}\right)\left(1 / P_{i-1}\right) c_{i}, \quad v_{i}=T_{i} S_{i} / Q_{i}, \quad w_{i}=\left(Q_{i} / S_{i}\right)\left(1 / P_{i}-1 / P_{i-1}\right) c_{i} .
$$

Note that $h\left(y_{i}, 0\right) \leqslant f\left(b_{i}\right) \leqslant h\left(y_{i}, 1\right)$ and $f\left(b_{i}\right)=h\left(y_{i},\left\lceil y_{i}\right]-y_{i}\right)$. A 'typical' function $h\left(y_{i}, r_{i}\right)$ is plotted in Fig. 3. It is shown there as a continuous function although it exists only when $b_{i}=y_{i} S_{i}$ is an integer. Since $S_{i}$ is an integer, an integer $y_{i}$ sets $b_{i}$ also to an integer.

In Fig. $3, y_{i}^{\mathrm{c}}$ indicates the minimum of the envelope $h\left(y_{i}, 0\right)$. It can be shown that, when $r_{i}=0$, the minimum of function (18) occurs at

$$
y_{i}^{\mathrm{c}}=\left(u_{i} / v_{i}\right)^{1 / 2}
$$

and that the optimum integer $y_{i}$ must be in the region $\left[\left\lfloor y_{i}^{c}\right],\left[y_{i}^{c}\right\rceil\right]$.

In order to find the optimum value of $b_{i}$ when $\left[y_{i}^{c}\right] S_{i} \geqslant Q_{i} / g_{i}$, we need only search for the lowest cost

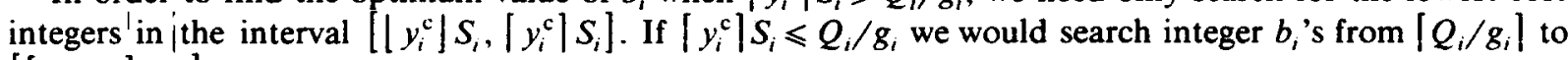
$\left.\left\lceil\mid Q_{i} / g_{i}\right\rceil / S_{i}\right\rceil S_{i}$.

Refinements to this procedure are possible. For instance, let $y_{i}^{*}$ be the value of $y$ that minimizes $h\left(y_{i},\left\{y_{i}\right\rceil-y_{i}\right)$, considered as a continuous function. Since $r_{i}=\left\lceil y_{i}^{\mathrm{c}}\right\rceil-y_{i}$, it can be shown from (18) that if $\left\lfloor y_{i}^{\mathrm{c}}\right\rfloor \leqslant y_{i}^{*} \leqslant\left\lceil y_{i}^{\mathrm{c}}\right]$ then $y_{i}^{*}$ is given by

$$
y_{i}^{*}=\left(\left(u_{i}+w_{i}\left[y_{i}^{\mathrm{c}}\right]\right) / v_{i}\right)^{1 / 2} \text {. }
$$

Only integer $b_{i}$ 's on either side of $y_{i}^{*} S_{i}$ would be checked if the constraint $b_{i} \geqslant Q_{i} / g_{i}$ did not interfere. However, unless $S_{i}$ values are very large such refinements are not necessary.

When $R_{i-1} \neq \hat{R}_{i-1}$ (note that this happens only when $j \neq\left(\left|Q_{i-1} / x_{i}\right|-1\right)$, expression (1) is used for $R_{i-1}$ and problem (16) becomes:

$$
\operatorname{minimize} \bar{f}\left(b_{i}\right)=\left(T_{i} / Q_{i}\right) b_{i}+c_{i} \max _{0 \leqslant j \leqslant b_{i}-1}\left\{j x_{i}\left(1 / P_{i}-1 / P_{i-1}\right)-\left\lfloor\frac{j x_{i}}{Q_{i-1}}\right\rfloor\left(1 / D-1 / P_{i-1}\right)\right\} .
$$

Let $b_{i}^{*}$ be the optimum number of batches in (16) and $b_{i}^{* *}$ the optimum number of batches in (21). Note that $\bar{f}\left(b_{i}^{*}\right)$ is an upper bound for $\bar{f}\left(b_{i}^{* *}\right)$.

As can be seen from (1), $R_{i-1} \geqslant x_{i} / P_{i}$; therefore

$$
\bar{f}\left(b_{i}\right) \geqslant\left(T_{i} / Q_{i}\right) b_{i}+\left(c_{i} Q_{i} / P_{i}\right) / b_{i} .
$$

We now search for $b_{i}^{* *}$ in the region of $\dot{f}\left(b_{i}\right)$ where

$$
\left(T_{i} / Q_{i}\right) b_{i}+\left(c_{i} Q_{i} / P_{i}\right) / b_{i} \leqslant \bar{f}\left(b_{i}^{*}\right) .
$$

The resulting range $\left[b_{i(\text { min })}^{* *}, b_{i(\max )}^{* *}\right]$ is obtained by setting the preceding inequality (22) to an equality and solving the resulting quadratic equation.

Now that the $S_{i}$ 's and $b_{i}$ 's are known, $Q_{1}$ may be updated to it's 'current-best' value as follows. Given $S_{1} \cdots S_{n}$ and $b_{1} \cdots b_{n}$ eq. (10) can be put in the form

$$
\mathrm{TC}=U / Q_{1}+V Q_{1}
$$

where

$$
\begin{aligned}
U= & D \sum_{i=1}^{n}\left(F_{i}+b_{i} T_{i}\right) /\left(S_{1} S_{2} \cdots S_{i-1}\right), \\
V \quad= & D \sum_{i=1}^{n} S_{1} S_{2} \cdots S_{i-1}\left\{\left(1 / D-1 / P_{i}\right)\left(c_{i}-c_{i+1}\right) / 2\right. \\
& \left.+\left[\delta_{i+1}\left(1 / P_{i+1}-1 / P_{i}\right)+\left(1-\delta_{i}\right) /\left(P_{i} b_{i}\right)+\delta_{i} /\left(P_{i-1} b_{i}\right)\right] c_{i}\right\}, \\
\delta_{n+1}= & 0 \text { and the } \delta_{i} \text { indicator variable is given in }(8) .
\end{aligned}
$$


The lot size that minimizes the total cost is expressed by $(U / V)^{1 / 2}$. Therefore, we can set the new $Q_{1}=\min \left(Q_{1 u},(U / V)^{1 / 2}\right)$ and the procedure is ready to loop again. It was found empirically that when $g_{i}$ 's are relatively small and binding on the value of $x_{i}$, the best value of $Q_{1}$ is often an integer multiple of some $g_{i}$. Therefore, in addition of the value of $Q_{1}$ found above, the closest such integer multiplies are checked. Since the cost is non-increasing and the number of possible $b_{i}$ 's is finite the procedure must converge.

\section{Computational example and conclusions}

Table 1 presents the problem parameters used in the example. The solution to the problem is shown in Table 2.

The first two sections of Table 2 show the solutions obtained by the heuristic procedure presented in this paper (without and with constraints). The total costs in each case are denoted by TC and the lower bounds on the costs are denoted by TC. lot-size model [5] when batch shipments are not allowed. The 'optimal' total cost here is denoted by TC*. For each of the cases ' $\Delta$ cost-ratio' indicates the percent cost in excess over the lower bound cost.

Note that the total costs in Table 2 for both the unconstrained and constrained cases are very close to the lower bound. Considering the fact that the lower bound on cost is a hypothetical result (i.e. very seldom attainable due to integrality requirements), the accuracy of the heuristic procedure is favourably reflected by the example. As a further test, 100 cases of each kind were computed with uniformly randomized input, the results of which are summarized in Table 3.

The results in Table 3 support confidence in the accuracy of the heuristic procedure. Except for very few cases, the $\Delta$ cost-ratio is very moderate. For the unconstrained problem 95 percent of the cases are 2.23 or less percent above the lower bound; for the constrained problem we found this percent to be 3.88 or less. This accuracy is especially remarkable if one considers that even for an optimal solution (for $Q_{i}=x_{i}$ ) 95 percent of the cases are 1.5 percent or less above the lower bound costs.

The computation of the heuristic procedure is rather efficient. Its time was between 0.16 and $0.22 \mathrm{CPU}$ seconds for a large sample of 12 stage cases on a CYBER computer. Experiments with very large numbers of stages, $10 \leqslant n \leqslant 300$, have shown that the computational time increases nearly proportionally with the number of stages up to $n=100$. For example, the following CPU seconds were needed (average of five problems) for some large numbers of stages: 0.41 for $n=50,0.98$ for $n=100,1.90$ for $n=150,4.15$ for $n=200$ and 6.02 for $n=300$.

Last but certainly not least, a noteworthy comparison can be made from Table 2 between the optimal total cost of a variable lot-size model $(\$ 15245.52)$ and the heuristic total cost $(\$ 12265.51)$ for the more flexible model presented in this paper. Note that both results include the same transportation cost per shipment whether it is a lot or a batch. The model which accommodates simultaneously both variable

Table 1

Problem parameters

\begin{tabular}{rllrlll}
\hline$i$ & $c_{i}$ & $P_{i}$ & $F_{i}$ & $T_{i}$ & $g_{i}$ & $L_{i}$ \\
\hline 1 & 2.5 & 250000 & 1.0 & 0.6 & 250 & 1500 \\
2 & 2.4 & 500000 & 6.0 & 0.6 & 250 & 1500 \\
3 & 2.2 & 375000 & 17.0 & 0.6 & 250 & 1500 \\
4 & 2.1 & 200000 & 8.0 & 2.2 & 250 & 1500 \\
5 & 1.7 & 150000 & 26.0 & 1.3 & 500 & 5000 \\
6 & 1.6 & 225000 & 9.0 & 3.2 & 500 & 5000 \\
7 & 1.5 & 275000 & 17.0 & 1.6 & 500 & 5000 \\
8 & 1.4 & 125000 & 24.0 & 1.0 & 500 & 5000 \\
9 & 0.6 & 175000 & 46.0 & 0.8 & 500 & 5000 \\
10 & 0.4 & 525000 & 18.0 & 5000 & 5000 \\
11 & 0.3 & 800000 & 16.0 & 8.0 & 6000 & $\infty$ \\
12 & 0.1 & 600000 & 60.0 & 5.8 & 6000 & $\infty$ \\
\hline
\end{tabular}

Number of stages: $n=12$; Demand rate: $D=60000$. 
[7] A.Z. Szendrovits, On the optimality of sub-batch sizes for a multi-stage EPQ model - a rejoinder. Management Sci. 23 (3) (1976) 334-338.

[8] A.Z. Szendrovits and Z. Drezner, Optimizing multi-stage production with constant lot size and varying numbers of batches, Omega 8 (6) (1980) 623-629.

[9] H.A. Taha and R.W. Skeith, The economic lot sizes in multi-stage production systems, AIIE Trans. 2 (2) (1970) $157-162$.

[10] H.M. Wagner, Research portfolio for inventory management and production planning systems, Operations Res. 28 (3) Part I (1980) 445-475. 